\title{
A New Gelling Agent and Rheology Modifier in Cosmetics: Caesalpinia spinosa Gum
}

\author{
Luigi Rigano ${ }^{1}$, Miriam Deola ${ }^{1}$, Francesca Zaccariotto ${ }^{2}$, Thomas Colleoni ${ }^{3}$ and \\ Nicola Lionetti ${ }^{1, * \mathbb{D}}$ \\ 1 Rigano Laboratories Srl, 20125 Milan, Italy; rigano@thecosmetologist.com (L.R.); \\ deola@thecosmetologist.com (M.D.) \\ 2 Department of Chemical and Pharmaceutical Sciences, University of Trieste, 34127 Trieste, Italy; \\ zaccariottofrancesca@gmail.com \\ 3 Silvateam Food Ingredients Srl, 24126 Bergamo, Italy; tcolleoni@silvateam.com \\ * Correspondence: lionetti@thecosmetologist.com
}

Received: 17 April 2019; Accepted: 31 May 2019; Published: 1 June 2019

\begin{abstract}
Caesalpinia spinosa gum is a vegetal polysaccharide obtained by grinding the endosperm of Caesalpinia spinosa seeds. It is commonly used as a rheology modifier in food industry. Its rheological behavior, compatibility with common cosmetic ingredients, and application as a thickener in different types of cosmetic formulations were investigated in this article. At low concentrations $(0.1-0.2 \%)$ the behavior is Newtonian; at higher percentages $(0.5-2.0 \%)$ it is pseudoplastic without thixotropy. The gum was tested in combination with salts, chelating agents, humectants, thickeners, pigments, nano UV filters, surfactants, conditioners, and ethanol, as well as in acidic/alkaline conditions. The wide compatibility and the interesting sensory profile, even in association with other thickeners, make the Caesalpinia spinosa gum a very promising ingredient for the thickening of various cosmetic products.
\end{abstract}

Keywords: rheology modifiers; Caesalpinia spinosa gum; galactomannans; thickeners; formulation

\section{Introduction}

The rheology modifier category, which comprises all the raw materials that significantly participate in the rheology of monophasic or biphasic systems, also includes ingredients called gelatinizers. These increase the viscosity of oils, aqueous solutions, suspensions, emulsions, and surfactants, often giving rise to systems with the thickness of a gel [1].

These substances might be classified according to their key application field, i.e. their use in hydrophilic, lipophilic, or surfactant systems. Alternatively, if the origin criteria are taken into account, they can be classified as natural, like guar, xanthan, and gellan gums or alginates [2], "natural-modified", for example cellulose derivatives (hydroxyethylcellulose, methylcellulose, hydroxypropylcellulose), synthetic, like products from the oil industry (acrylates, polyesters, waxes), or finally mineral, like clays and pyrogenic silica. As current market trends require greener, sustainable, and safe ingredients, the importance of natural ingredients is on the rise in the rheology modifiers field. Indeed, they have some limitations, such as, for instance, their high sensitivity to extreme $\mathrm{pH}$ or high ionic concentrations and their relative susceptibility to microbial and environmental contamination. The non-perfect reproducibility of the gelatinized system could be an additional drawback. Natural polymers usually show more or less branched polysaccharide backbones which are quite hydrophilic, and the gel formation is strictly related to their chemical structure and concentration, typically similar to carbohydrates [3,4]. 


\section{Caesalpinia spinosa Gum}

Caesalpinia spinosa gum, also known as Peruvian carob, is a natural gum obtained by grinding the endosperm of Caesalpinia spinosa seeds. Caesalpinia spinosa is a plant belonging to the Leguminosae family, native to the Peruvian and Bolivian Andes [5].

Caesalpinia spinosa flour (Figure 1), an odorless white to ivory-colored powder, has been to date been largely used in the food industry as a thickening agent. For this reason, it is internationally recognized as completely safe for other human applications.

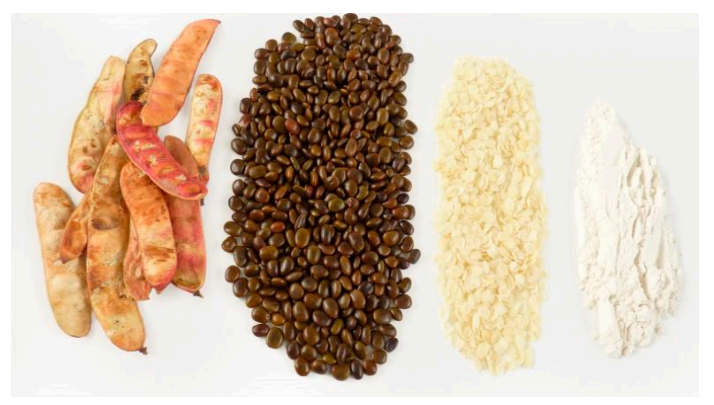

Figure 1. Caesalpinia spinosa gum, from the pod to the flour.

Chemically speaking, Caesalpinia spinosa gum is a polysaccharide composed of (1-4)- $\beta$-D-mannopyranose linear chains, branched through (1-6) bonds with $\alpha$-D-galactopyranose units in a 3:1 ratio (Figure 2), a characteristic structure resembling that of other natural gums such as guar and carob, which, on the other hand, show a different mannose to galactose ratio [6,7].

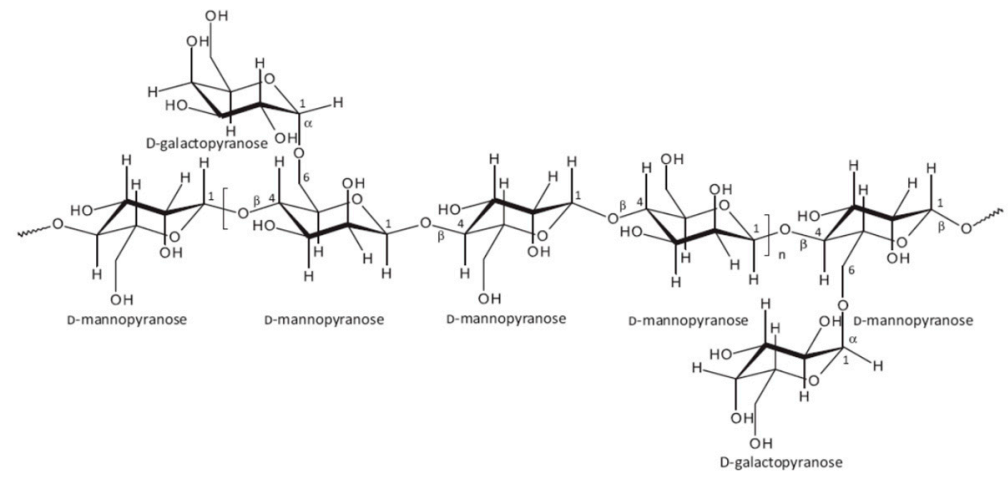

Figure 2. Chemical structure of Caesalpinia spinosa gum.

The dispersibility in water of these polymers is proportional to the percentage of galactopyranose units in the polysaccharide chains [8]. Caesalpinia spinosa gum, with $25 \%$ galactose (meaning a medium branching level), results partially soluble in cold water, while it easily dissolves in hot water, fully hydrating while forming a medium force gel of exceptional softness. The $\mathrm{pH}$ value of an aqueous solution of Caesalpinia spinosa gum at $2 \%$ is 6.4 at $25^{\circ} \mathrm{C}$.

\section{Materials and Methods}

\subsection{Rheological and Viscosity Measurements}

Aqueous solutions of one grade of Caesalpinia spinosa gum (Biosericum, Silvateam) at 0.1\%, $0.2 \%$, $0.5 \%, 1 \%$, and $2 \%$ were prepared by homogenizing the powder in deionized water with a high shear mixer (Silverson L4R, Silverson Machines Ltd, Waterside, Chesham HP5 1PQ, UK) at $65{ }^{\circ} \mathrm{C}$.

Rheological measurements were performed by using a rotational rheometer (Discovery HR-1Rheostress RS150, TA Instruments, New Castle, USA) at $25{ }^{\circ} \mathrm{C}$. 
Viscosity measurements were carried out with a rotational viscometer (Brookfield DV-I+, Brookfield Engineering Laboratories, Inc.

11 Commerce Boulevard, Middleboro, MA 02346 U.S.A.) at different concentrations $(0.1 \%, 0.2 \%$, $0.5 \%, 1 \%, 2 \%)$ at $25^{\circ} \mathrm{C}$ and at different temperatures $\left(25^{\circ} \mathrm{C}, 35^{\circ} \mathrm{C}, 45^{\circ} \mathrm{C}, 55^{\circ} \mathrm{C}\right)$ at $1 \%$.

\subsection{Compatibility Trials}

Compatibility trials were carried out by adding several common cosmetic ingredients to the aqueous solution of the gum at $1 \%$. The samples were checked visually and their viscosity was measured immediately after the preparation, then after 24 and 48 hours.

- $\quad$ Salts: $\mathrm{NaCl}$ and $\mathrm{MgSO}_{4}$ up to $2 \%$ concentration.

- Chelating agents such as disodium and tetrasodium salts of ethylenediaminotetraacetic acid $(0.1 \%)$, sodium salts of ethylenediamine disuccinic and glutamic-diacetic acids $(0.4 \%)$.

- Hydrotropes: betaine, glycerin, isopentyldiol, propylene glycol, and 1,3-propandiol up to 5\%.

- $\quad$ Ethanol up to $40 \%$

- $\quad$ Lactic acid up to $\mathrm{pH} 3$ and $\mathrm{NaOH}$ up to $\mathrm{pH} 12$.

- $\quad$ Five common natural or synthetic thickeners were added to the aqueous solution of Caesalpinia spinosa gum at $0.5 \%$ by homogenizing: xanthan gum at $0.5 \%$, carrageenan at $1 \%$, carbomer at $0.5 \%$, hydroxyethylcellulose at $1 \%$, and the copolymer ammonium acryloyldimethyltaurate/VP at $0.5 \%$. Sensory evaluations of the gels were carried out by a panel of trained experts.

The compatibility of the gum and its influence on the foam height and quality were tested with the following common surfactants: sodium laureth sulfate (SLES), disodium cocoamphodiacetate, disodium laureth sulfosuccinate, cocamidopropyl betaine, and coco-glucoside. Foam height was measured by preparing an aqueous solution of each surfactant at $0.5 \%(100 \mathrm{~mL}$ in a $400-\mathrm{mL}$ beaker); the solution was homogenized with a high shear mixer (Silverson L4R,) equipped with a crown for emulsions with circular holes during $1 \mathrm{~min}$. Then the foam was poured slowly in a 250-mL cylinder with an internal diameter of $35 \mathrm{~mm}$. The foam height was measured with a ruler in millimeters (mm), $1 \mathrm{~min}$ and $5 \mathrm{~min}$ after preparation. The quality was checked visually and with in-use trials. The compatibility of Caesalpinia spinosa gum aqueous solution at $0.25 \%, 0.5 \%$, and $1 \%$ was tested with each mentioned surfactant at Washing Active Substance - WAS 15. Then, blends made of sodium lauryl ether sulfate as main surfactant at $10 \%$ + secondary surfactant (disodium cocoamphodiacetate or disodium laureth sulfosuccinate or cocamidopropyl betaine or coco-glucoside) at $2 \%$ and $4 \%$ were tested in association with the aqueous solution of the gum at $0.5 \%$. A visual analysis and viscosity measurements were performed immediately after the preparation, and after 24 and 48 hours.

Then, $1 \%$ cetrimonium chloride was added to the aqueous solution of the gum at $0.5 \%$ to test their compatibility. The sample was checked visually and its viscosity was measured immediately after the preparation, and after 24 and 48 hours.

Additional trials were carried out in order to investigate the compatibility with make-up pigments and the ability to keep them in suspension. The following inorganic solids were added to an aqueous solution of Caesalpinia spinosa gum at $1 \%$ : titanium dioxide $10 \%$, zinc oxide $5 \%$, brown iron oxide 5\% (CI 77499, CI 77491, CI 77492), and D\&C Red n.7 Calcium Lake (CI 15850) 2\% by homogenizing with a high shear mixer (Silverson L4R). The dispersions were evaluated visually and by an optical microscope (Optika M-699, magnitude 600×,

OPTIKA Srl, Via Rigla, 30 - 24010 Ponteranica, Italy) just after the preparation and after 24/48 h.

Moreover, nano UV filters, i.e. uncoated zinc oxide at $25 \%$ and silica-coated titanium dioxide at $20 \%$ were added to an aqueous solution of the gum at $1 \%$ by homogenizing. The ease of dispersion during production and the appearance of the samples after $24 / 48 \mathrm{~h}$ were checked. 


\subsection{Suspending and Emulsification Power}

In order to verify the suspending power of some inorganic solids, even in comparison with xanthan gum, dispersions of the two gums at $1 \%$ with $25 \%$ micronized $\mathrm{ZnOor} 10 \% \mathrm{TiO}_{2}$ and at $0.2 \%$ with $10 \% \mathrm{TiO}_{2}$ were prepared and centrifuged at different speeds (1000, 3000, $\left.5000 \mathrm{rpm}\right)$ for $5 \mathrm{~min}$ at $25^{\circ} \mathrm{C}$ (Heraeus centrifuge, Multifuge 1-SR, Thermo Fisher Scientific, 3rd Avenue, Waltham, MA, USA).

Finally, an additional investigation was carried out to define the potential emulsification power of the gum. The assessment was performed by adding increasing amounts $(0.3 \%, 0.6 \%, 0.9 \%$, $1.2 \%, 3 \%$ ) of an oil blend of medium polarity (composed of caprylic/capric triglyceride, dicaprylyl ether, hydrogenated polydecene $1: 1: 1$ ) to Caesalpinia spinosa gum solution at $1 \%$ by homogenizing at maximum speed for $5 \mathrm{~min}$ (Silverson L4R,). The samples were evaluated visually and by optical microscopic observation (Optika M-699, magnitude 600×) measuring the average diameter of the oil drops (five measurements for each dispersion) immediately after the preparation, after 48 hours and one week.

\section{Results and Discussion}

\subsection{Rheological and Viscosity Measurements}

From a rheology point of view, very dilute Caesalpinia spinosa gum aqueous solutions (0.1-0.2\%) show an almost pure Newtonian behavior (Figure 3). In other words, viscosity does not change when increasing the shear force. As concentrations progressively increase, the polymer solution acquires pseudoplastic characteristics and its viscosity decreases when increasing the shear force.

Flow Curves

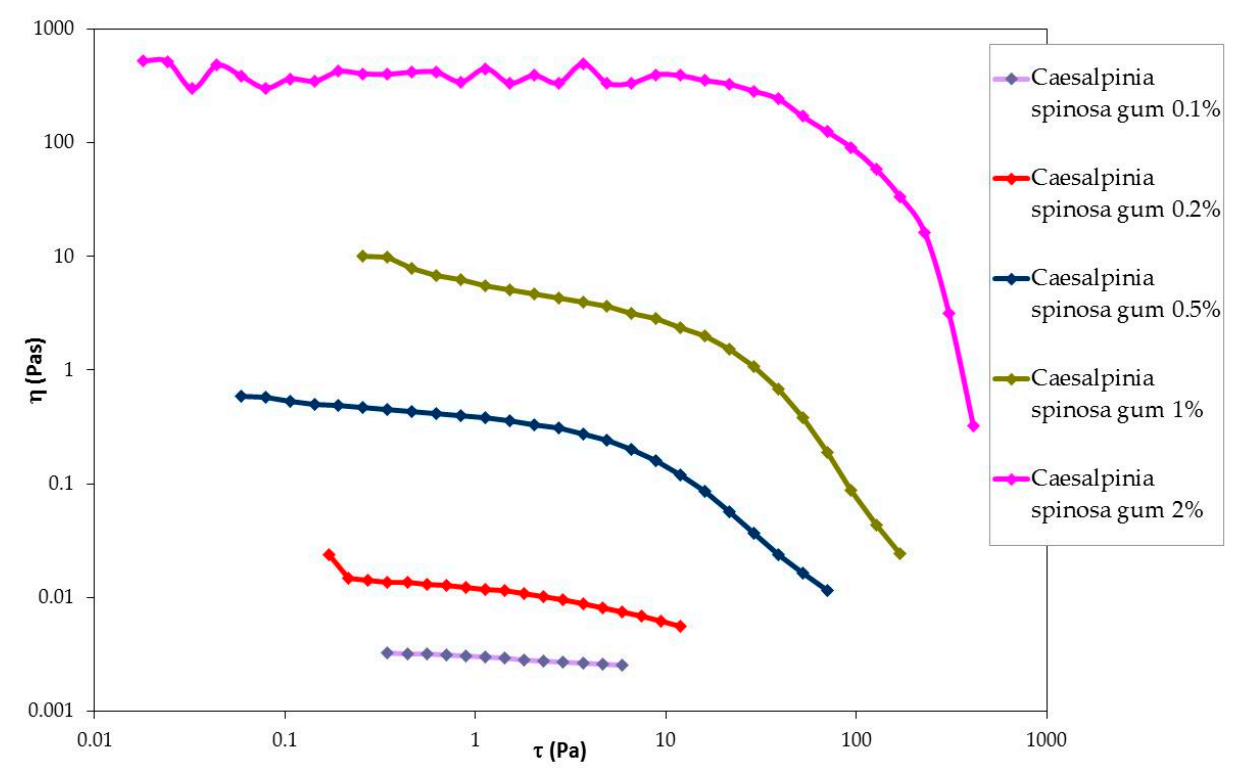

Figure 3. Flow curves of Caesalpinia spinosa gum at several concentrations $(0.1 \%, 0.2 \% 0.5 \%, 1 \%, 2 \%)$ (Cone and plate rheometer, at $25^{\circ} \mathrm{C}$ ).

At high concentrations, such as $2 \%$, its behavior becomes viscoelastic. Indeed, the polymer keeps a constant viscosity at increasing shear forces, up to a threshold value, where it takes on a properly pseudoplastic behavior.

In general, aqueous solutions of Caesalpinia spinosa gum do not exhibit thixotropic characteristics, and therefore their structure-rebuilding time is comparable to the initial part of the rheological path (Figure 4). It is important to underline that viscosity is inversely proportional to temperature (Figure 5), as occurs frequently. 


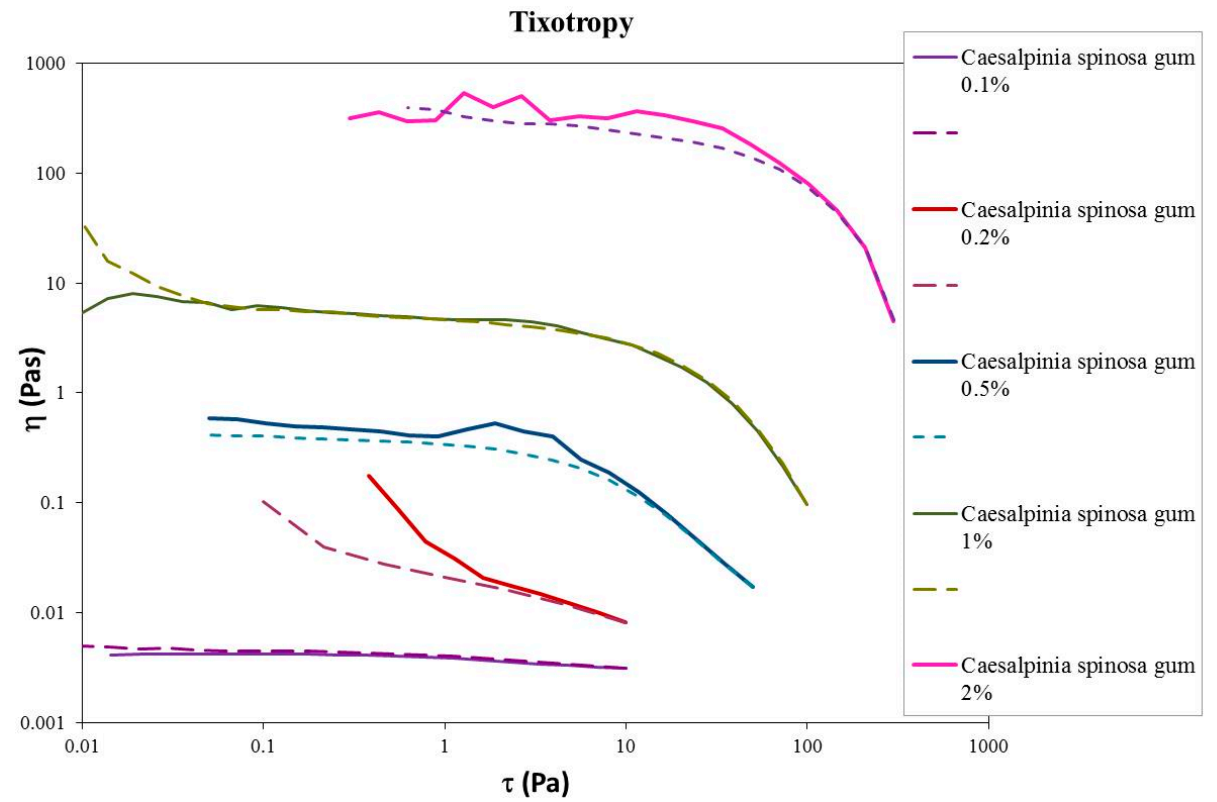

Figure 4. Caesalpinia spinosa gum thixotropy at several concentrations $(0.1 \%, 0.2 \% 0.5 \%, 1 \%, 2 \%)$. Dotted lines refer to the return path.

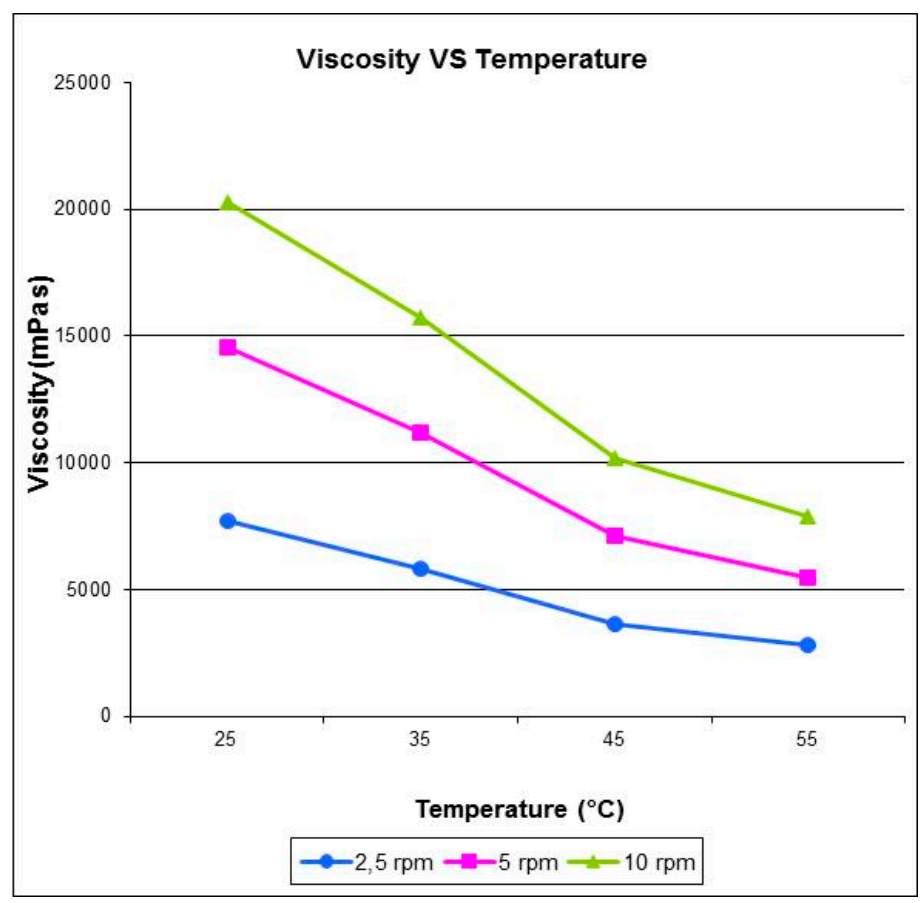

Figure 5. Caesalpinia spinosa gum viscosity at different temperatures.

Table 1 and Figure 6 show the relationship between viscosity and concentration. An important increase in viscosity is observed from the $1 \%$ concentration. 
Table 1. Viscosity values for each Caesalpinia spinosa gum concentration.

\begin{tabular}{|c|c|c|c|c|}
\hline \multirow{2}{*}{$\begin{array}{c}\text { Caesalpinia spinosa } \\
\text { gum (\%) }\end{array}$} & \multicolumn{4}{|c|}{$\begin{array}{c}\text { Viscosity (mPa.s) } \\
\text { Brookfield DV-I+, } 25{ }^{\circ} \mathrm{C}\end{array}$} \\
\hline & $2.5 \mathrm{rpm}$ & $5 \mathrm{rpm}$ & $10 \mathrm{rpm}$ & $20 \mathrm{rpm}$ \\
\hline 0.10 & 4 & 6 & 10 & 12 \\
\hline 0.20 & 12 & 16 & 20 & 22 \\
\hline 0.50 & 720 & 696 & 648 & 576 \\
\hline 1.00 & 8320 & 7360 & 6120 & 4740 \\
\hline 2.00 & 131,000 & 98,400 & 69,200 & 44,800 \\
\hline
\end{tabular}

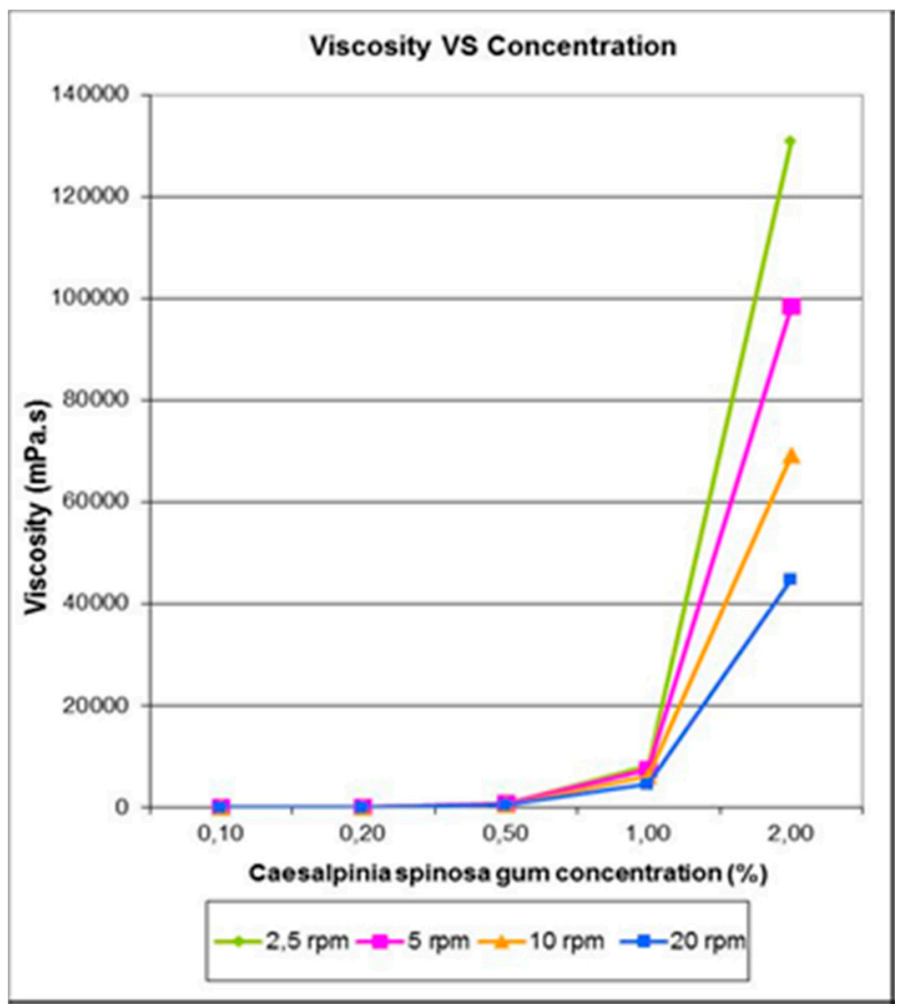

Figure 6. Caesalpinia spinosa gum viscosity at different concentrations.

\subsection{Compatibility Trials}

This grade of Caesalpinia spinosa gum showed an excellent compatibility with most ingredients at concentrations usually adopted in cosmetics (such as sodium chloride and magnesium sulfate), salts commonly used to thicken cleansing formulae or to stabilize water in oil (W/O) emulsions, and chelating agents like sodium salts of ethylenediaminotetraacetic, ethylenediamine disuccinic, and glutamic-diacetic acids. In fact, the appearance of the samples did not change after $48 \mathrm{~h}$ and the viscosity slightly increased.

Hydrotropes, such as betaine, glycerin, isopentyldiol, propylene glycol, and 1,3-propandiol showed perfect compatibility and gave a slightly higher viscosity to aqueous dispersions. In addition, by preliminary wetting the gum with the fluid hydrotopes, the dispersion in water is easier, therefore facilitating the swelling procedure.

Caesalpinia spinosa gum proved to be stable in a $\mathrm{pH}$ range from 3 to 12 , without changes in appearance nor viscosity. Moreover, it tolerated the addition of ethanol up to $10 \%$, even if the viscosity underwent a significant increase. 
Caesalpinia spinosa gum showed in general good compatibility with the natural and synthetic rheology modifiers tested, i.e., carrageenan, carbomer, hydroxyethylcellulose, and ammonium acryloyldimethyltaurate/VP copolymer, with a strong viscosity increase. Additionally, there was an interesting synergic effect with xanthan gum $[9,10]$.

Indeed, the combination of the two polymers forms instantly a compact gel, sharing this characteristic with other galactomannans, unlike what happens when they are used alone [11].

Moreover, Caesalpinia spinosa gum enhanced the sensorial properties of the binary gels in terms of softness, spreading, thickness, velvety after-feel, and lower stickiness.

The foam height of surfactants solutions tends to decrease when adding Caesalpinia spinosa gum, even if good foam stabilization is evident. The gum proved to be compatible at $0.25 \%$ with disodium cocamphodiacetate (WAS 15) and with sodium laureth sulfate (WAS 15) up to $1 \%$, showing in this case an increase in viscosity. As far as the blends are concerned, the gum at $0.5 \%$ resulted compatible with SLES $10 \%$ + coco-glucoside up to $4 \%$ and SLES $10 \%$ + disodium laureth sulfosuccinate up to $4 \%$, showing an increase in viscosity in both cases, and with SLES $10 \%$ + cocamidopropyl betaine at $2 \%$, showing a decrease in viscosity. The benefits imparted to surfactants by Caesalpinia spinosa gum, such as improvement of foam quality, a thicker soft feeling during massaging, and the high safety level, make it ideal for delicate cleansing, for example in personal and baby hygiene and cleansing recipes for intolerant and hypersensitive skins. In addition, the gum proved to be compatible with the conditioner cetyltrimethylammonium chloride, making it suitable for thickening hair products, from cleansers to conditioning masks.

In the case of solid dispersions, Caesalpinia spinosa gum demonstrated good compatibility with the pigments used in make-up formulations, such as iron oxides, titanium dioxide, and zinc oxide, except the Calcium Lake, for which dispersion was difficult and not homogeneous. Even inorganic micronized UV filters like uncoated $\mathrm{ZnO}$ and silica-coated $\mathrm{TiO}_{2}$ proved to be compatible with this gum.

\subsection{Suspending and Emulsification Power}

Caesalpinia spinosa gum showed good suspending power of some inorganic powders at $1 \%$, slightly more than xanthan gum. In fact, centrifuged samples of dispersions of both gums at $1 \%$ with nano $\mathrm{ZnO} 25 \%$ or $\mathrm{TiO} 210 \%$ did not show any precipitate at $1000 \mathrm{rpm}$. In contrast, at 3000 and $5000 \mathrm{rpm}$ they presented some precipitate, higher in case of xanthan gum, in particular with $\mathrm{ZnO}$. Decreasing the percentage of the gums to $0.2 \%$ by dispersing $10 \% \mathrm{TiO}_{2}$ the samples did not show any precipitate at $1000 \mathrm{rpm}$, similar amounts of precipitate at $3000 \mathrm{rpm}$, and a higher amount only in the case of Caesalpinia spinosa gum at $5000 \mathrm{rpm}$. However, it is important to underline that the difference in viscosity of the two gums at $0.2 \%$ influences the suspending properties (viscosity, Brookfield DV-I+, $25^{\circ} \mathrm{C}$, Spindle 2, $2.5 \mathrm{rpm}$ : xanthan gum $1,200 \mathrm{mPa} \cdot \mathrm{s}$, C. spinosa gum $\left.12 \mathrm{mPa} \cdot \mathrm{s}\right)$.

Trials carried out by dispersing an oil blend into Caesalpinia spinosa gum aqueous solutions at $1 \%$ showed some oil emulsifying properties up to an oil concentration of about $1 \%$. In fact, the average diameter of the oil droplets just after the preparation was $3.8 \mu \mathrm{m}$ at $0.3 \%$ oil, $10.3 \mu \mathrm{m}$ at $0.9 \%$ oil, and $20.0 \mu \mathrm{m}$ at $3 \%$ oil. After one week, the values kept quite constant: $4.2 \mu \mathrm{m}$ at $0.3 \%$ oil, $10.2 \mu \mathrm{m}$ at $0.9 \%$ oil, and $21.8 \mu \mathrm{m}$ at $3 \%$ oil. This characteristic, alongside its verified suspending power, makes Caesalpinia spinosa gum a good alternative to xanthan gum as a thickening and stabilizing agent.

\subsection{Optimal Concentration of Usage}

The right concentration of Caesalpinia spinosa gum to maximize its suspending properties, while avoiding any "gommage" effect after spreading the product over the skin, ranges between $0.1 \%$ and $1 \%$. Indeed, the film forming properties become predominant above this concentration, even though they might be needed in the preparation of make-up products with long-lasting characteristics, such as eyeliners and mascaras or face masks and muds. 


\section{Formulation Examples}

\begin{tabular}{|c|c|c|}
\hline Phase & Ingredients (INCI* name) & $\% \mathrm{w} / \mathrm{w}$ \\
\hline \multirow{6}{*}{ A } & Aqua & to 100 \\
\hline & Allantoin & $0.10-0.30$ \\
\hline & Xylitol & $2.00-5.00$ \\
\hline & Zinc PCA & $0.05-0.15$ \\
\hline & Panthenol & $0.20-0.60$ \\
\hline & Trisodium Ethylenediamine Disuccinate, Aqua & 0.30 \\
\hline A1 & Carbomer & $0.30-0.50$ \\
\hline \multirow{2}{*}{ A2 } & Isopentyldiol & $3.00-6.00$ \\
\hline & Caesalpinia Spinosa Gum & $0.30-0.50$ \\
\hline \multirow{5}{*}{ B } & Phenoxyethanol & 0.80 \\
\hline & o-Cymen-5-ol & 0.10 \\
\hline & Parfum & $0.10-0.30$ \\
\hline & Menthol, Menthyl Lactate & $0.08-0.12$ \\
\hline & PEG-40 Hydrogenated Castor Oil & $0.80-1.20$ \\
\hline \multirow[t]{2}{*}{$\mathbf{E}$} & Triethanolamine & qs to $\mathrm{pH} 6$ \\
\hline & & 100.00 \\
\hline
\end{tabular}

* INCI = International Nomenclature of Cosmetic Ingredients

Production technology:

1. Prepare phase A by mixing until complete solution.

2. Disperse A1 into phase A by homogenizing at room temperature.

3. Prepare phase $\mathrm{A} 2$ and add it to phase A while homogenizing.

4. Prepare phase $B$ and add it into the main mixer while homogenizing.

5. Adjust $\mathrm{pH}$ to 6 by adding phase $\mathrm{E}$ and homogenize for $5 \mathrm{~min}$.

Specifications:

pH: 6.0

Viscosity: (Brookfield DV-I+; S05; $25^{\circ} \mathrm{C}, \mathrm{t}=24 \mathrm{~h}, \mathrm{mPa} \cdot \mathrm{s}$ )

- $\quad 2.5 \mathrm{rpm}: 92,480$

- $\quad 5.0 \mathrm{rpm}: 56,000$

- $\quad 10.0$ rpm: 33,880

\section{Appearance: Transparent Gel}

This formula shows the compatibility of Caesalpinia spinosa gum with common water-soluble additives of a different chemical nature. This good compatibility is also reported when blending Caesalpinia spinosa gum with other hydrophilic polymers. The result is an improvement of the sensorial properties of the end product, imparting softness and freshness. 
Discipline hair mask.

\begin{tabular}{|c|c|c|}
\hline Phase & Ingredients (INCI name) & $\% \mathrm{~W} / \mathrm{W}$ \\
\hline A & Aqua & to 100 \\
\hline A1 & Polyquaternium-10 & $0.05-0.15$ \\
\hline A2 & Caesalpinia Spinosa Gum & $0.80-1.20$ \\
\hline \multirow{6}{*}{ B } & Behentrimonium Methosulfate, cetearyl alcohol & $3.00-6.00$ \\
\hline & C12-13 Lactate & $2.00-4.00$ \\
\hline & Isostearyl Alcohol & $0.50-1.50$ \\
\hline & Cetrimonium Chloride & $0.50-1.50$ \\
\hline & Stearamidopropyl Dimethylamine & $0.20-0.60$ \\
\hline & Butyrospermum Parkii Butter & $1.00-3.00$ \\
\hline C & Aqua & $20.00-30.00$ \\
\hline D & Glycerin & $2.00-6.00$ \\
\hline $\mathbf{E}$ & Phenoxyethanol & 0.60 \\
\hline $\mathbf{F}$ & Amodimethicone, Cetrimonium Chloride, Trideceth-10 & $2.00-6.00$ \\
\hline G & Aminopropyl Dimethicone & $0.50-1.50$ \\
\hline $\mathbf{H}$ & Parfum & $0.20-0.50$ \\
\hline I & Sodium Hydroxide, Aqua & $0.20-0.40$ \\
\hline $\mathbf{L}$ & Lactic Acid, Aqua & $0.20-0.40$ \\
\hline $\mathbf{M}$ & Sodium Benzoate, Potassium Sorbate, Aqua & 0.35 \\
\hline & & 100.00 \\
\hline
\end{tabular}

Production technology:

1. Add phase A in the main mixer, then firstly add phase A1 and secondly add phase A2, while homogenizing at room temperature.

2. Heat up at $70^{\circ} \mathrm{C}$ while homogenizing.

3. Prepare phase $\mathrm{B}$ in a side mixer and heat up to $70^{\circ} \mathrm{C}$.

4. Slowly add phase B into phase A, while homogenizing.

5. Add phase $\mathrm{C}$ gradually, while homogenizing.

6. Add phases D-H, homogenizing after each addition.

7. Add phases I-M, mixing after each addition.

8. Cool down to room temperature while mixing.

Specifications:

pH: 4.5

Viscosity: (Brookfield DV-I +; S06; $25^{\circ} \mathrm{C}, \mathrm{t}=24 \mathrm{~h}, \mathrm{mPa} \cdot \mathrm{s}$ )

- $\quad 2.5$ rpm: 118,000

- $\quad 5.0 \mathrm{rpm}: 72,600$

- $\quad 10.0$ rpm: 43,800 
Appearance: White Thick Emulsion

This formulation scheme shows the compatibility of Caesalpinia spinosa gum with different types of monomeric and polymeric cationic conditioners, silicones and a vegetal butter. At high concentrations, Caesalpinia spinosa gum provides a thick and firm gel structure, suitable for hair masks.

Oil/Water $(\mathrm{O} / \mathrm{W})$ foundation

\begin{tabular}{|c|c|c|}
\hline Phase & Ingredients (INCI name) & $\% \mathrm{~W} / \mathrm{W}$ \\
\hline \multirow{3}{*}{ A } & Aqua & to 100 \\
\hline & Disodium EDTA & 0.05 \\
\hline & Betaine & $0.50-1.50$ \\
\hline \multirow{3}{*}{ A1 } & Propylene Glycol & $1.00-3.00$ \\
\hline & Glycerin & $4.00-8.00$ \\
\hline & Caesalpinia Spinosa Gum & $0.20-0.60$ \\
\hline A2 & Glycerin, Inulin Lauryl Carbamate & $1.00-3.00$ \\
\hline \multirow{8}{*}{ B } & Hydrogenated Polydecene & $2.00-5.00$ \\
\hline & Butylene Glycol Dicaprylate/Dicaprate & $5.00-10.00$ \\
\hline & Caprylic/Capric Triglyceride & $5.00-8.00$ \\
\hline & Isoamyl Laurate & $2.00-6.00$ \\
\hline & Limnanthes Alba Seed Oil, Butyrospermum Pparkii Butter Extract & $2.00-6.00$ \\
\hline & Sorbitan Stearate & $0.50-2.00$ \\
\hline & Glyceryl Stearate & $1.00-2.50$ \\
\hline & BHT & 0.05 \\
\hline \multirow{3}{*}{ B1 } & CI 77891 & $8.00-12.00$ \\
\hline & CI 77492 & $1.00-2.00$ \\
\hline & CI 77491, CI 77492, CI 77499 & $1.00-2.00$ \\
\hline $\mathrm{C}$ & Phenoxyethanol, Ethylhexylglycerin & 1.00 \\
\hline \multirow[t]{2}{*}{$\mathbf{D}$} & Parfum & $0.10-0.30$ \\
\hline & & 100.00 \\
\hline
\end{tabular}

Production technology:

1. After preparing phase A and phase A1 separately, slowly add phase A1 into phase A and homogenize.

2. Add phase $\mathrm{A} 2$ in the main mixer, heat up to $60^{\circ} \mathrm{C}$ and homogenize again.

3. Prepare phase $\mathrm{B}$ in a side melting-vessel and heat up to $70^{\circ} \mathrm{C}$.

4. Add phase B1 pigments, previously mixed and milled, to the fluid B phase, while homogenizing.

5. Add slowly phase B to phase A while homogenizing.

6. Cool to room temperature.

7. Add phase $\mathrm{C}$ and phase $\mathrm{D}$, then homogenize.

Specifications:

pH: 5.7

Viscosity: (Brookfield DV-I+; S04; $25^{\circ} \mathrm{C}, \mathrm{t}=24 \mathrm{~h}, \mathrm{mPa} \cdot \mathrm{s}$ )

- $\quad 2.5 \mathrm{rpm}: 28,480$

- $\quad 5.0$ rpm: 19,600 
- $\quad 10.0$ rpm: 13,160

Appearance: Skin-Colored Fluid Emulsion

This example demonstrates the compatibility of Caesalpinia spinosa gum with mineral pigments, favoring a long-lasting hiding and mat effect on the skin surface. It also shows the compatibility of Caesalpinia spinosa gum with other emulsifying polysaccharides and its stabilization power, even at low emulsifier concentration.

Personal hygiene cleanser.

\begin{tabular}{ccc}
\hline Phase & Ingredients (INCI name) & \% W/W \\
\hline \multirow{2}{*}{ A } & Aqua & to 100 \\
\cline { 2 - 3 } & Panthenol & $0.20-0.60$ \\
\cline { 2 - 3 } & Codium Benzoate & 0.25 \\
\cline { 2 - 3 } & Trisodium Ethylenediamine Disuccinate, Aqua & $0.50-1.00$ \\
\hline & Phenoxyethanol & 0.30 \\
\hline & Phenethyl Alcohol & 0.80 \\
\hline & Coco Glucoside, Aqua & 0.10 \\
\hline C & Sodium Laureth Sulfate, Aqua & $2.00-5.00$ \\
\hline D & Disodium Laureth Sulfosuccinate, Aqua & $3.00-6.00$ \\
\hline & Lactic Acid, Aqua & $2.00-5.00$ \\
\hline
\end{tabular}

Production technology:

1. Prepare phase A while homogenizing, then heat up to $60{ }^{\circ} \mathrm{C}$.

2. Cool down below $40^{\circ} \mathrm{C}$, then add phase $\mathrm{B}$ while homogenizing.

3. Add phase $\mathrm{C}$, while mixing.

4. Use phase $\mathrm{D}$ to adjust $\mathrm{pH}$ to a value between 4.5 and 4.8 .

Specifications:

pH: 4.7

Viscosity: (Brookfield DV-I + ; S02; $25^{\circ} \mathrm{C}, \mathrm{t}=0, \mathrm{mPa} \cdot \mathrm{s}$ )

- $\quad 2.5 \mathrm{rpm}: 3760$

- $\quad 5.0$ rpm: 3360

- $\quad 10.0$ rpm: 2836

Appearance: Slightly Opaque Fluid

This example shows the compatibility of Caesalpinia spinosa gum with surfactants, a very useful property to create delicate cleansing formulas. Using Caesalpinia spinosa gum, the foam is softer, the bubbles are finer, and after the application the skin is smooth. 
Toothpaste, sensitive gums

\begin{tabular}{|c|c|c|}
\hline Phase & Ingredients (INCI Name) & $\% \mathrm{~W} / \mathrm{W}$ \\
\hline \multirow{6}{*}{$\mathbf{A}$} & Aqua & to 100 \\
\hline & Sodium Saccharin & $0.15-0.30$ \\
\hline & Sodium Monofluorophosphate & $0.60-0.80$ \\
\hline & Sorbitol & $25.00-35.00$ \\
\hline & PEG-400 & $2.00-6.00$ \\
\hline & Hydroxyacetophenone & 0.20 \\
\hline A1 & Caesalpinia Spinosa Gum & $0.30-0.80$ \\
\hline \multirow{2}{*}{ B } & Aqua & $2.00-6.00$ \\
\hline & Titanium Dioxide & $0.50-1.50$ \\
\hline \multirow{3}{*}{$\mathrm{C}$} & Hydrated Silica & $6.00-12.00$ \\
\hline & Hydrated Silica & $8.00-14.00$ \\
\hline & Silica & $1.00-3.00$ \\
\hline \multirow{2}{*}{ D } & Aroma & $1.00-1.50$ \\
\hline & CI 19140, CI 42090 & $0.03-0.06$ \\
\hline \multirow{2}{*}{ E } & Aqua & $2.00-8.00$ \\
\hline & Sodium Lauryl Sulfate & $1.00-1.50$ \\
\hline \multirow[t]{2}{*}{$\mathbf{F}$} & Sodium hydroxide, Aqua & $0.50-1.00$ \\
\hline & & 100.00 \\
\hline
\end{tabular}

Production technology:

1. Prepare phase A, adding each ingredient separately, then heat up to $60^{\circ} \mathrm{C}$, while stirring

2. Disperse Caesalpinia spinosa gum (A1), while homogenizing.

3. Pour phase $B$ into phase $A$ while homogenizing, then add phase $C$ and homogenize again.

4. Cool down below $40^{\circ} \mathrm{C}$, then add phase $\mathrm{D}$, while homogenizing.

5. Add phase $\mathrm{E}$, while mixing.

6. Cool down to room temperature, then adjust $\mathrm{pH}$ using phase $\mathrm{F}$.

Specifications:

pH: 6.8

Viscosity: (Brookfield RVT, Helipath-D; $25^{\circ} \mathrm{C}, \mathrm{t}=24 \mathrm{~h}, \mathrm{mPa} \cdot \mathrm{s}$ ):

- $\quad 2.5$ rpm: 328,000

- $\quad 5.0$ rpm: 188,000

- $\quad 10.0 \mathrm{rpm}: 100,000$

\section{Appearance: Light-Green Paste}

Caesalpinia spinosa gum can be easily added in any toothpaste formulation, being extremely compatible with abrasive agents such as hydrated silica. Caesalpinia spinosa gum gives the product a soft perception while tooth brushing, a useful property especially in the case of sensitive gums.

\section{Conclusions}

The application data presented above highlight the strengths of a grade of Caesalpinia spinosa gum as a novel rheology modifier for the cosmetic industry.

First of all, the good compatibility with a large amount of other common cosmetic ingredients enables a wide range of possible formulations. 
Secondly, the synergy of Caesalpinia spinosa gum with xanthan gum is useful to create peculiar products such as jellies without the need to use large concentrations of the two thickeners.

Moreover, among the various properties, those of most impact are those linked to the enhancement of sensorial properties of finished products in terms of softer and richer skin-feel and velvety after-feel, completely free of any sticky perception.

Finally, other important key features of Caesalpinia spinosa gum include its low cost, its easiness of dispersion especially when pre-wet in glycols, and the fact that it is an extremely safe ingredient. These are all good reasons for its widespread use within the food industry. These characteristics make Caesalpinia spinosa gum an added-value ingredient within modern cosmetic formulation strategies, requiring, now more than ever, natural, safe, sustainable, economic, and versatile solutions.

Author Contributions: Conceptualization, L.R.; Investigation, M.D. and F.Z.; Data Curation, M.D.; Writing-Original Draft Preparation, M.D. and F.Z.; Writing-Review \& Editing, L.R. and N.L.; Supervision, L.R. and T.C.

Conflicts of Interest: All authors Luigi Rigano, Miriam Deola, Francesca Zaccariotto, Thomas Colleoni and Nicola Lionetti declare no conflict of interest.

\section{References}

1. Braun, D.B.; Rosen, M.R. Preface. In Rheology Modifiers Handbook-Practical Use and Application; William Andrew Publishing: New York, NY, USA, 2000; Available online: https://epdf.pub/rheology-modifiershandbook-practical-use-and-applilcation-materials-and-process.html (accessed on 31 May 2019).

2. Burey, P.; Bhandari, B.R.; Howes, T.; Gidley, M.J. Hydrocolloid Gel Particles: Formation, Characterization, and Application. Food Sci. Nutr. 2008, 48, 361-377. [CrossRef] [PubMed]

3. D'Agostinis, G.; Mignini, E. Il Manuale del Cosmetologo, 2nd ed.; Tecniche Nuove: Milano, Italy, 2014; pp. 51-53.

4. Ramawat, K.G.; Mérillon, J.-M. Polysaccharides: Bioactivity and Biotechnology; Springer: Berlin/Heidelberg, Germany, 2015; pp. 1867-1892.

5. Santos, M.B.; dos Santos, C.H.C.; de Carvalho, M.G.; de Carvalho, C.W.P.; Garcia-Rojas, E.E. Physicochemical, thermal and rheological properties of synthesized carboxymethyl tara gum (Caesalpinia spinosa). Int. J. Biol. Macromol. 2019, 134, 595-603. [CrossRef] [PubMed]

6. Wu, Y.; Ding, W.; He, Q. Molecular characteristics of tara galactomannans: Effect of degradation with hydrogen peroxide. Int. J. Food Prop. 2017, 20, 3014-3022. [CrossRef]

7. Wu, Y.; Ding, W.; Jia, L.; He, Q. The rheological properties of tara gum (Caesalpinia spinosa). Food Chem. 2015, 168, 366-371. [CrossRef] [PubMed]

8. Prajapati, V.D.; Jani, G.K.; Moradiya, N.G.; Randeria, N.P.; Nagar, B.J.; Naikwadi, N.N.; Variya, B.C. Galactomannan: A versatile biodegradable seed polysaccharide. Int. J. Biol. Macromol. 2013, 60, 83-92. [CrossRef] [PubMed]

9. Wu, Y.; Ding, W.; He, Q. The gelation properties of tara gum blended with $\mathrm{k}$-carrageenan or xanthan. Food Hydrocoll. 2018, 77, 764-771. [CrossRef]

10. Grisel, M.; Aguni, Y.; Renou, F.; Malhiac, C. Impact of fine structure of galactomannans on their interactions with xanthan: Two co-existing mechanisms to explain the synergy. Food Hydrocoll. 2015, 51, 449-458. [CrossRef]

11. Bresolin, T.M.B.; Milas, M.; Rinaudo, M.; Reicher, F.; Ganter, J.L.M.S. Role of galactomannan composition on the binary gel formation with xanthan. Int. J. Biol. Macromol. 1999, 26, 225-231. [CrossRef]

(C) 2019 by the authors. Licensee MDPI, Basel, Switzerland. This article is an open access article distributed under the terms and conditions of the Creative Commons Attribution (CC BY) license (http://creativecommons.org/licenses/by/4.0/). 